\title{
Desafíos axiológicos para la psiquiatría contemporánea. Pluralismo e imperativo bioético
}

\author{
Fernando Lolas ${ }^{1}$
}

Editorial

\section{EL PARADIGMA TECNOLÓGICO Y LA PSIQUIATRÍA}

El gran desafío de la psiquiatría contemporánea no es solamente técnico, es, además, moral. ${ }^{1}$

Esta aseveración requiere clarificaciones. Se ha impuesto la idea de que el paradigma técnico, que asimila el trabajo en salud mental a lo que ocurre en otras áreas técnicas de la medicina, es el único que puede prometer progreso indefinido, mejorar la estimación social de las profesiones y aumentar su capacidad de servir. ${ }^{2}$

El pensamiento progresista es tan ubicuo como el desarrollismo. El desarrollo socioeconómico y su necesidad es manifestación del progresismo. ${ }^{3}$ En último análisis, ambas posturas desplazan hacia un incierto futuro la superación de todas las limitaciones. Prometen bienestar y satisfacciones para todos, sin importar su origen, condición o posibilidades. La medicina, como panacea, será un bien del cual gozarán todas las personas gracias al desarrollo de nuevos productos y técnicas. Lo nuevo significa en el ideario consumista siempre lo mejor, lo que se necesita, lo que trae felicidad. La reinterpretación de la historia que ello supone debe ser materia de debate. Los avances deben examinarse no solamente a la luz de lo que traen o prometen sino de lo que es socialmente eficaz. El mercado es un creador de necesidades y la racionalidad tecnocrática tiene como fin presentar soluciones para problemas aún no sentidos como tales. La medicina no solamente debe aliviar nuestros males. Debe hacernos más bellos, inteligentes, longevos. Debe responder no solamente a las necesidades. También debe satisfacer deseos. La medicina del deseo es la culminación del arte. Esta ideología está presente en los escritos técnicos de modo implícito.

Las profesiones ganan ascendiente social gracias a sus mitos. Medicalizar los trastornos del comportamiento, es- pecialmente los graves, sirvió para humanizar. La tradición que atribuye a Philippe Pinel la liberación de los enfermos mentales de sus cadenas en la Francia de 1793, es sin duda interpretable como un triunfo de los ideales revolucionarios. ${ }^{4}$ Retrospectivamente, se considera un respaldo emocional a la igualdad de los seres humanos y una demostración del ideal de servicio. A la vuelta de unas décadas, los críticos señalan que el poder de la profesión psiquiátrica ha servido y sirve para consolidar estructuras de poder y fundarlas en el argumento de lo patológico..$^{5}$ Aun la "cura moral" de los alienistas del siglo XIX, la fundación del asilo para segregar el crimen, la locura y la pobreza, o las propuestas tecnocráticas para su prevención y curación, pueden interpretarse bajo esta óptica. La impresión de que se superan etapas hacia una auténtica medicina de la persona, aunque refutada cuando se analiza la situación global, sirve de argumento para fines muy diversos. La industria farmacéutica, aliada a la profesión psiquiátrica, la convierte en su instrumento. Puede proclamar, con razón, que sus desarrollos son beneficiosos. Pero también sirve a sus propios intereses económicos. De hecho, más de algún avance conceptual, como la definición de ciertos comportamientos como patológicos y necesitados de tratamiento, se deben a que existen formas de abordarlos antes insospechadas.

El paradigma tecnológico, y sus consiguientes intereses, pueden tener consecuencias distintas de las esperadas. Por ejemplo, es discutible que solamente la introducción de la terapia neuroléptica haya hecho innecesarios los asilos. Su reducción tuvo que ver, además, con consideraciones sociales y económicas. Y la desinstitucionalización realizada en muchos países, por ejemplo en Italia, partió como ideología humanitaria, se basó probablemente en una desmesurada población asilar debida a razones distintas del diagnóstico (pobreza, por ejemplo) y ha conducido en algunos casos a formas soterradas de abuso y discriminación. Muchos años

Centro Interdisciplinario de Estudios en Bioética, Instituto de Estudios Internacionales y Clínica Psiquiátrica Universitaria, Universidad de Chile.

Miembro del Comité Internacional de SALUD MENTAL.

Correspondencia: Dr. Fernando Lolas. Diagonal Paraguay 265, Of. 806, Santiago, Chile. E-mail: flolas@u.uchile.cl 
después que Franco Basaglia reconociera que el manicomio no era fruto de la ciencia sino del poder, el argumento sigue vigente. Algunos ex pacientes se llaman a sí mismos "sobrevivientes". Pese a los significativos progresos de la industria y la investigación biomédica sigue existiendo desconfianza y percepción de que la situación no ha cambiado. No solamente porque en nombre de la ciencia que subyace a la psiquiatría técnica se abusa de ella con fines políticos (y no solamente en la antigua Unión Soviética o la Alemania nazi). También, porque los beneficios no llegan a todos los que los necesitan. Cuando las organizaciones internacionales proclaman que no hay salud sin salud mental, su propuesta consiste en aumentar el número de profesionales científicamente entrenados. ${ }^{6}$ Cuando una disciplina como la psiquiatría revisa cada cierto tiempo sus criterios diagnósticos y terapéuticos manifiesta que su misma falibilidad se usa para incrementar su ámbito de poder en nombre de la ciencia. Lo demuestran las sucesivas ediciones de los DSM, que incluyen progresivamente más categorías susceptibles de considerarse patologías. Es una manera de incrementar poder mediante argumentos que manifiestan otros intereses. El modelo de pensamiento hegemónico en las naciones civilizadas confunde la comprensión de otras culturas con pedir que su modo de argumentar e intervenir sea universal. El mismo concepto de salud mental global, pese a su auge, tiene a veces la impronta del pensamiento colonialista implícito en la noción de "salud internacional". En más de una dimensión recuerda que la antropología clásica distinguía entre "ellos" y "nosotros". "Ellos" eran quienes tienen otro sistema de creencias, sin duda menos valioso y en necesidad de una intervención civilizatoria. Los sistemas diagnósticos, cuya misión esencial es "rotular" y "etiquetar", introducen una cisura y no mejoran la "alienación" que significa ser diferente.

El paradigma tecnológico se basa en algunos supuestos. Por ejemplo, que la biología humana es universal. Un cerebro es igual a otro cerebro. Pensamiento igualitario sin duda. Sin embargo, ignora que la "mente no está en el cerebro". Es una construcción social y cultural. Afirmar que un antidepresivo resuelve los problemas humanos o que la industria de la psicoterapia es esencial para el bienestar impide reflexionar. Hacerlo significa poner en duda los beneficios de la ciencia y la tecnología. Ningún experto puede dudar de aquello que constituye el fundamento de su aprecio social. Debemos preconizar el uso extendido de medicamentos milagrosos y confiar en su eficacia. Debemos sostener que solamente el modelo hegemónico de pensamiento es el único que brinda soluciones a los problemas de las personas. La salud pública, entendida como el esfuerzo organizado de una comunidad para prevenir enfermedades y promover la salud, debe prescribir y proscribir. Indicar aquello necesario para el bienestar e impedir lo que lo daña. La salud, en tanto concepto valórico, tiene faz unívoca y lo sano es lo que dictan los cánones de la ciencia occidental.
Comprobamos que no se extirpa la violencia ni se mejora la aceptación social de lo diferente con estos desarrollos. Se obtura la comprensión y se la suplanta por el poder. Por otro lado, estimulan la aparición de movimientos alternativos radicales, aprovechados por charlatanes y comerciantes para atacar a la profesión médica o satanizar a la industria. Situación que, lejos de resolver el problema, lo profundiza. Destruye la posibilidad de renovar. Desplaza el interés por resolver los desafíos hacia contiendas estériles sobre "creencias" y "datos". Quizá uno de los escollos reside en que los valores y creencias de las personas son declarados trabas para el ejercicio de una vocación indiscutible de servicio. Sin considerar que una adecuada interpretación, aunque parezca alejada de las demandas inmediatas, sería garantía de una mayor satisfacción por parte de profesionales y usuarios. Una correcta interpretación debe considerar que todas las profesiones se encuentran en una interfaz entre política, economía y tecnología. ${ }^{7}$ El objeto de las profesiones, su tarea, debe considerarse a la luz de sus medios y sus fines. La misma construcción social "trastorno mental" debe mucho a prejuicios, influencias no científicas y poder de rotular. Sin necesidad de pasar a las filas de la "antipsiquiatría" o de condenar acríticamente la racionalidad económica es necesario plantear el asunto desde un punto de vista amplio. Éste radica en examinar los fundamentos morales del arte. No solamente por sus fines y medios. También porque en su forma más accesible involucra la disposición a la deliberación y el diálogo.

\section{PLURALISMO METÓDICO Y PLURALISMO AXIOLÓGICO}

Caracterizan a la psiquiatría -usando esta denominación para incluir todo acto o intervención relacionados con la salud mental y el comportamiento- un pluralismo metódico y un pluralismo axiológico. Antes hemos aludido a esto como heterogeneidad, para incluir también los puntos de vista de quienes piden y quienes dan ayuda y las diferentes perspectivas que ello implica. ${ }^{8}$

Pluralismo metódico, pues las fuentes de información son variadas. La tríada psicofisiológica incluye la conducta manifiesta, la ideación y la fisiología. Ninguno de estos ámbitos se reproduce fielmente en el otro. Cada uno es un texto y los otros dos sirven de con-texto. Esto es, se interpreta mejor una expresión facial si se la relaciona con la ideación concomitante. Una señal fisiológica adquiere una significación diferente si se conoce lo que las personas dicen o hacen. La disociación entre estos ámbitos indica cuán estéril es inferir uno partiendo de otro. El lenguaje empleado y los métodos para recoger información son diferentes. El conjunto de los tres, unido al conocimiento de la historia individual y colectiva (plasmada en recuerdos o en producciones objetivas) permite describir síndromes, emociones o estados y procesos patológicos. La socialización profesional, que constituye 
especialistas y expertos, separa los universos discursivos y la interpretación de los problemas individuales y sociales.

Una consecuencia de esta pluralidad de fuentes de información, y su diferente impacto en la descripción y la formulación de diagnósticos, es la existencia de lenguajes parcialmente irreconciliables. Se forman comunidades de expertos cuya socialización dificulta el entendimiento con otros expertos. Eventualmente, puede llevar a una fisiología sin mente y a una mente sin cerebro, posturas extremas que recuerdan el viejo "escuelismo" de las tradiciones inmaduras.

Paralelo a este pluralismo metódico debe considerarse el pluralismo axiológico. En sus diversas formas, como investigación o intervención, hay numerosos valores involucrados en la psiquiatría. Por valor se entiende la perfección ideal que da sentido a lo deseado u observado.

Así, hay valores económicos como la eficiencia, la competitividad y la honestidad. También, valores técnicos. Y finalmente valores morales. Puede sostenerse que los valores no morales son instrumentales y que los realmente inherentes a la condición humana son fines en sí mismos. Así, la dignidad, la autonomía o la no-maleficencia, pueden considerarse esenciales en el trato interhumano, por ser valores humanos y no por ser instrumentos para conseguir otros fines.

A menudo se supone, en la literatura bioética, que cuando se habla de autonomía se quiere significar lo que la tradición filosófica consideró tal. El concepto kantiano de autonomía no es equiparable a la libérrima posibilidad de elegir con que a menudo se lo confunde. Supone que se actúa conforme a la razón. Cuando hoy se invoca la autonomía en las numerosas variantes del consentimiento informado no solamente se alude a un valor moral. En realidad, tal como lo entiende la mayoría de los profesionales, es un conjuro ritual que evita problemas legales o hace concesiones a las limitadas elecciones a que se enfrentan los pacientes o los sujetos de investigación. En tal sentido, el concepto autonomía tiene, en el ideario del sentido común, valor tanto moral como técnico. Es, en realidad, un "concepto puente" entre las obligaciones morales y las necesidades técnicas. Otro tanto puede decirse de la beneficencia, la maleficencia o la justicia, entendidas en el ámbito del sentido común. Basta examinar los procesos deliberativos de los comités de ética o las prescripciones contenidas en las pautas bioéticas convencionales para percibir su calidad híbrida, moral y técnica. En rigor, la adopción de tales conceptos no se ha debido a convicciones éticas sino a conveniencias impuestas por el cumplimiento de estándares y el acceso a recursos. Las revistas especializadas no publican estudios que no cumplan, al menos formalmente, los requisitos contenidos en las normas usuales para la investigación clínica. Su adopción no implica compromiso moral sino adaptación a exigencias a veces no sentidas por los investigadores como esenciales.

La ética es la explicitación y fundamentación de la moralidad común a través del lenguaje. Éste puede ser descrip- tivo o prescriptivo. Es innegable la influencia de la cultura y la costumbre en su estructuración concreta. Una comunidad que no valora la autonomía individual o la restringe para algunos de sus miembros (lo que se liga a la capacidad y la competencia) no es idéntica a otra que la erige como supremo valor. En el campo de las intervenciones terapéuticas, la ambigüedad suele no estar ausente. Respetar todo lo que los pacientes desean contradice el papel de experto que se atribuye al profesional. A veces, también, contraviene la norma legal.

En la jerarquía de las instancias de decisión ética podemos hablar de valores, principios, normas. Quizá los principios, que median entre valores y normas, tengan precisamente el carácter híbrido que les hemos atribuido. Por estar entre los valores y las normas de comportamiento amalgaman y sirven de puente entre los comportamientos concretos (guiados por imperativos técnicos, políticos, económicos) y los valores (el "deber ser" ideal). Esto es, nuestra observación de que incluyen dimensiones morales y no morales es justamente su mayor importancia. Aunque no siempre sea percibido así. Muchas de las personas, aun las ilustradas, creen obvio suponer que la tradición filosófica les ayudará a aplicar principios en la vida diaria. La ética es, en este sentido, una semántica de los términos que el lenguaje común usa en sentido valorativo. En esa estimación (la ética es, en palabras de Ortega, una estimativa) el interpretar correctamente lo que otros dicen, en el ámbito de su particular universo moral, es esencial. La perspectiva hermenéutica, la interpretación de otros textos, es inseparable de la consideración ética en el contexto del diálogo.

Si algo ha cambiado en las últimas décadas es la comprobación de que la deliberación y el diálogo fundan y fundamentan el comportamiento moral sobre la base de intercambios entre personas. ${ }^{9}$ Así como se suele distinguir entre eficacia y efectividad, una el efecto en condiciones ideales $\mathrm{y}$ otra en condiciones reales, nunca las situaciones que se encuentra pueden ser esquematizadas nítidamente. No hay blancos y negros, solamente grises, en la realidad. Ello no justifica un relativismo del tipo "todo vale". Pero es necesario recordar las enseñanzas de la casuística, que siempre tomaba (y toma) en cuenta la circunstancia (circum-stare, lo que rodea) para emitir valoración y juicio. Las éticas monológicas, derivadas con rigor de un sistema filosófico, por muy ingenioso o atractivo que sea, no consideran siempre lo que en religión suele llamarse "el signo de los tiempos". Spinoza, con su ética "more geometrico", no podía anticipar los conocimientos de la tecnociencia contemporánea. Ni Kant predecir que la "paz perpetua" sería una utopía para la postIlustración irracional. Si la ética filosófica hubiera ayudado, o ayudara, a bien-vivir y al bien-estar más allá de los confines de un individuo, no habría guerras ni conflictos.

La medicina es el arte por excelencia de la interpersonalidad al servicio de las necesidades humanas. ${ }^{10}$ No por mera compasión, pues ya decía el gran clínico Osler, regius professor de medicina, que la equanimitas -el distanciamiento que 
no enturbia el juicio- era una virtud del médico que sabe su oficio. Quien se acerca al experto en busca de ayuda -para curar, sanar o ser comprendido- desea la comprensión, pero también exige el diagnóstico certero, la prescripción adecuada, el pronóstico acertado. Quiere el ingenio fecundo, el juicio profundo y el gusto rotundamente jocundo, como decía Baltasar Gracián. El arte de saber ejercer el arte, la vieja virtud de la prudencia (la phrónesis) es el sentido de adoptar el paradigma bioético. Nada agrega en términos cuantitativos o mensurables. Sí aporta la cualidad de lo humanamente permisible, aquello que, más allá de la comprensión y la explicación (Verstehen y Erklären, las dimensiones que Jaspers distinguía) no enturbian la destreza y no obnubilan el juicio.

\section{EL IMPERATIVO BIOÉTICO}

Cuando Fritz Jahr, pionero en la articulación semántica de la obligación moral hecha precepto, hablaba del imperativo bioético lo hacía desde la óptica que respeta la vida en todas sus formas. ${ }^{11} \mathrm{Si}$ se reemplaza la palabra vida por salud, se tiene la versión médica de ese imperativo universal. La presciencia de Jahr relativizaba este precepto agregando "en la medida de lo posible". Que se convierta en auténtico elemento integrador del arte, y no en su mero complemento, es la tarea de quienes trabajan generando conceptos y aplicando técnicas al servicio de otros. Nadie puede hacer lo imposible. Quizá el mayor mérito de los clásicos principios de la bioética estadounidense -autonomía, beneficencia, nomaleficencia y justicia, el "mantra de Georgetown"- sea precisamente que no son solamente principios filosóficos sino incluyen, y no de manera accesoria, el imperativo técnico. Pues el "bien hacer", el saber-hacer y el saber-estar en la dignidad del oficio, es tanto imperativo moral como técnico. Y quien ignora su oficio ya actúa de manera inmoral si reclama las recompensas sociales que demandan los oficios -el prestigio, el dinero, el poder y el amor.

Una de las enseñanzas prácticas que se derivan de este punto de vista es que lo axiológico -en la pluralidad de sus manifestaciones, morales y no morales- no puede ser una imposición desde fuera. No puede la racionalidad filosófica suplantar la racionalidad de los conocedores del oficio. Solamente quien se confronta con la cotidianidad de los desafíos reales, con las demandas del mundo, puede tener la palabra para ordenar, prescribir o prohibir. ${ }^{12}$

Una consideración importante se refiere al papel de la deliberación axiológica. Tanto en lo moral como en lo técnico, el desafío no consiste en reparar yerros sino en anticipar problemas. Normalmente se reacciona frente a errores. La postura proactiva de una ética consecuente con la contemporaneidad consiste en anticiparlos y evitarlos. El papel de la ética no debe ser solamente compensatorio sino anticipatorio. Ello supone conocer la historia y extraer enseñanzas del pasado. Conocer las formas que ha adoptado la inves- tigación y la práctica, estudiar sus contextos culturales y reconocer las formas en que sus cultores han aprendido y actuado es el mejor modo de articular el imperativo bioético del diálogo. Pues no se dialoga sólo con los contemporáneos. También con el pasado y con el futuro, lo que exige una forma de imaginación moral que puede aprenderse y debe cultivarse.

La aportación más decisiva en la reorientación paradigmática que significa la bioética para la medicina y la psiquiatría es la consideración del diálogo como instrumento para la preceptiva prudente. La deliberación debe incluir todas las perspectivas y puntos de vista, pues lo real es aquello que iluminan muchas miradas. En el campo de los oficios de ayuda, las profesiones que tratan con personas deben considerar como su finalidad el que lo que se considere mejoría o progreso sea tal para todos los interesados ("stakeholders"). Así, por ejemplo, aunque la práctica de los comités de ética suele incorporar la opinión de especialistas y no especialistas, lo que se considera un buen "resultado" no siempre incluye la opinión de los segundos. Sugerencias en tal sentido son de considerar. ${ }^{13}$ Respetar la vida, el imperativo bioético de Fritz Jahr, es equivalente a respetar la salud. Y de salud, como de belleza, hay muchas formas merecedoras de respeto. El desafío axiológico es también un desafío de competencia intercultural. ${ }^{14,15}$

\section{REFERENCIAS}

1. Lolas F. Ethics in psychiatry: a framework. World Psychiatry 2006;5:185187.

2. Bracken $P$, Thomas $P$, Timini $S$ et al. Psychiatry beyond the current paradigm. Brit J Psychiat 2012;201:430-434.

3. Dupas G. O Mito do progresso. Sao Paulo: Editora UNESP; 2006.

4. Pichot P. Zum "Mythos Pinel". Nervenarzt 2002;73:301-302.

5. Foucault, M. El poder psiquiátrico. México: Fondo de Cultura Económica; 2005.

6. Saxena S, Funk M, Chisholm D. WHO's mental health action plan 2013-2020: what can psychiatrists do to facilitate its implementation? World Psychiatry 2014;13:107-109.

7. Lolas F. Medical praxis: an interface between ethics, politics, and technology. Soc Sci Med 1994;39:1-5.

8. Lolas F. El discurso de la psiquiatría. Acta Psiquiat Psicol Amer Lat (Buenos Aires) 1994;40:80-82.

9. Lolas F. Bioethics and psychiatry: a challenging future. World Psychiatry 2002;1:123-124.

10. Lolas F. La medicina como ciencia de acciones: Consecuencias para la psiquiatría. Acta Luso Españolas Neurología Psiquiatría 1990;18:29-33.

11. Lolas F. Bioethics and animal research. A personal perspective and a note on the contribution of Fritz Jahr. Biological Research (Santiago) 2008;41:119-123.

12. Lolas F. Psychiatry: medical specialty or specialized profession? World Psychiatry 2010;9:34-35.

13. Thorncrof G, Slade M. New trends in assessing the outcomes of mental health interventions. World Psychiatry 2014;13:118-124.

14. Fernando GA. The roads less traveled: Mapping some pathways on the global mental health research roadmap. Transcultural Psychiatry 2012;49:396-417.

15. Lolas F. Salud mental global como bien público: sugerencias para la reflexión ética. Rev Neuropsiquiatr (Lima) 2013;76:131-136. 